\title{
Solvent Exchange in Liquid Methanol and Rate Theory
}

\author{
Liem X. Dang and Gregory K. Schenter \\ Molecular Theory Group \\ Physical Sciences Division \\ Pacific Northwest National Laboratory
}




\begin{abstract}
To enhance our understanding of the solvent exchange mechanism in liquid methanol, we report a systematic study of this process using molecular dynamics simulations. We use transition state theory, the Impey-Madden-McDonald method, the reactive flux method, and Grote-Hynes theory to compute the rate constants for this process. Solvent coupling was found to dominate, resulting in a significantly small transmission coefficient. We predict a positive activation volume for the methanol exchange process. The essential features of the dynamics of the system as well as the pressure dependence are recovered from a Generalized Langevin Equation description of the dynamics. We find that the dynamics and response to anharmonicity can be decomposed into two time regimes, one corresponding to short time response $(<0.1 \mathrm{ps})$

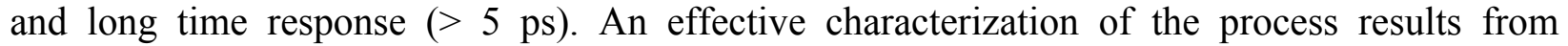
launching dynamics from the planar hypersurface corresponding to Grote-Hynes theory. This results in improved numerical convergence of correlation functions.
\end{abstract}




\section{Introduction}

The study of the thermodynamic and kinetic properties of solvent exchange provides molecular-level detail about the subtle balance between solvent-solute and solvent-solvent interaction that advance understanding of solvation phenomena in general. Because of the significance of exchange in physical processes, this phenomenon has been the subject of considerable efforts in recent years.[1-5] Minor differences in the properties of solvents or solutes can cause major changes in their role in physical and chemical properties. The reactivity of a solvent requires rearrangement of the hydration shell, which involves the replacement of solvent molecules from first hydration shell. This process, also known as a solvent-exchange

reaction, is associated with activation volume, $\Delta \mathrm{V}^{\ddagger}$, which is a key indicator of the solventexchange mechanism.[6-8]

In this article, we describe molecular dynamics (MD) simulation studies performed with solvent molecules treated explicitly using empirical polarizable potential models. This approach allows us to examine solvent and polarization effects on the computed properties and their influence on the mechanism and rate of the exchange process. We present our rate theory results on the solvent-exchange mechanism in liquid methanol, requiring the characterization of the potentials of mean force (PMF) and friction kernels, resulting in rate constants obtained using transition-state theory (TST).[9] The Impey-Madden-McDonald method (IMM),[10] the reactive flux (RF) method,[11] and Grote-Hynes $(\mathrm{GH})$ theory treatments of the dynamic response of the solvent[12] are compared. Pressure effects on these aqueous systems are of particular interest because of their relevance to the development of activation volume concepts. The current developments are naturally extended to the understanding of the influence of nuclear quantum mechanical effects on the kinetic properties of aqueous solutions. Our primary goal in this work 
is to develop a molecular model that describes the thermodynamics and the kinetics of the solvent-exchange process in liquid methanol. To complete this activity, we computed PMFs that include polarization effects, and then, we estimated the rate constant using TST. Next, we used the RF method to calculate the time dependence of the reactive flux correlation function and the corresponding transmission coefficients, $\kappa_{R F}$. We also carry out a simulation using IMM to compute the residence time and then the corresponding $\kappa_{I M M}$. Finally, we use GH theory to compute the transmission coefficients, $\kappa_{G H}$, for this exchange process. Subsequently, we

evaluate the pressure dependence of rate constants and then computed the corresponding $\Delta \mathrm{V}^{\ddagger}$ for the exchange process. In an effort to understand the nature of the dynamical recrossing and the limitation of GH theory rate estimates, we explore the consequences of detailed Generalized Langevin Equation (GLE) dynamics associated with this system.

This current work differs from those earlier efforts in that we include polarization effects into potential models. Characterization of this influence on the rate constant results and $\Delta \mathrm{V}^{\ddagger}$ is the primary focus of this work. The remainder of our paper is organized as follows. In Section 2, the potential models and simulation methods are described. Results and discussion are presented in Section 3, and our conclusions are presented in Section 4.

\section{Simulations and Methods}

\section{A. Rate Constants}

Here we provide some important aspects of the methodology used in our study. The TST involves no explicit dynamics, but requires a good reaction coordinate.[13] TST can be corrected by using explicit dynamics. This is the strength of the RF method, although it's convergence can be challenging.[11] The GH method provides the exact analytical result for a harmonic GLE system.[12,14] IMM requires a direct sampling of population dynamics, but the reactant and 
product states are defined by a residence time, $t^{*}$ instead of a spatial criteria alone. This leads to numerically converged residence times that are sensitive to the choice of $t^{*} \cdot[5,10]$

a. The TST

Using the computed PMFs, we compute the TST rate constants using Eq. 1. Here, $r^{*}$ is the position of the barrier top, and $\mu$ is the solvent reduced mass.

$$
k^{T S T}=\sqrt{\frac{k_{b} T}{2 \pi \mu}} \frac{\left(r^{*}\right)^{2} \exp \left(\frac{W\left(r^{*}\right)}{k_{b} T}\right)}{\int_{0}^{r^{*}} r^{2} \exp \left(\frac{W(r)}{k_{b} T}\right) d r}
$$

The variational aspect of TST is well established.[15] TST overestimates the values of rate constants because it assumes that, once the dynamics of reactive species reach the transition state, they always proceed to the products side of the reaction. The actual rate constant accounting for re-crossings resulting from the solvent dynamics is given by $k=\kappa k^{T S T}$ where $\kappa$ is the transmission coefficient, which gives the probability of successful exchanges at the transition state. We computed the transmission coefficients using three different methods: 1) GH theory, 2) the RF method, and 3) the IMM approach.

b. The GH theory

In the GH theory, the transmission coefficient $\kappa_{G H}$ is obtained by iteratively solving Eq. 2.

$$
\kappa_{G H}=\left(\kappa_{G H}+\int_{0}^{\infty} d t \frac{\zeta(t)}{\omega_{b}} e^{-\omega_{b} \kappa_{G H} t}\right)^{-1}
$$

The GH theory transmission coefficient involves the frequency component of the time dependent friction coefficient, $\zeta(t)$, at the Laplace frequency, $\omega_{b} \kappa_{G H}$, relevant in the barrier region. In this equation, $\zeta(t)$ is the time-dependent friction kernel acting on the reaction coordinate, and $\omega_{b}$ is 
the barrier frequency determined through parabolic approximation to the PMF at the barrier region. The time-dependent $\zeta(t)$ acting on the reaction coordinate at $r^{*}$, is computed using Eqs. 3 and 4.

$\zeta(t)=\frac{1}{\mu k_{b} T}\left\langle R\left(t, r^{*}\right) \cdot R\left(0, r^{*}\right)\right\rangle$

$R(t, r)=F(t, r)-\langle F(t, r)\rangle$

where, $r^{*}$ is the position of the barrier maximum, $\mu$ is the reduced mass, $k_{b}$ is the Boltzmann constant, and $T$ is the temperature. $R(t, r)$ is fluctuation of the solvent force exerted on the reaction coordinate axis, which is the distance between the center of masses of two methanol molecules.

c. The RF method

The transmission coefficient using the RF method is obtained from the plateau value of the time-dependent transmission coefficient $\kappa_{\mathrm{RF}}(t)$ :

$\kappa_{\mathrm{RF}}(t)=\frac{<(v \cdot \hat{r}) \delta\left(r-r^{\dagger}\right) \theta\left(r(t)-r^{\dagger}\right)>}{<(v \cdot \hat{r}) \delta\left(r-r^{\dagger}\right) \theta(v \cdot \hat{r})>}$

To determine $\kappa_{\mathrm{RF}}(t)$, the methanol system has to start at the transition state indicated by $\delta\left(r-r^{\dagger}\right)$, followed by a time evolution in either the forward or backward direction $(r(t)$ or $r(-t))$. The term $v \cdot \hat{r}$ represents the initial methanol velocity and $\theta(v \cdot \hat{r})$ is the Heaviside function to account for only positive velocities.

\section{B. IMM Method}

The IMM method allows the residence time of a solvent molecule in the first solvation shell of another molecule to be calculated. It is determined from the normalized time-correlation 
function of for the population of methanol molecules in the first solvation shell of the methanol, as given in Eq. 6.

$P(t)=<n_{\alpha}\left(t_{i}, t_{f}, t^{*}\right)>/<n_{\alpha}\left(t_{i}, t_{i}, t^{*}\right)>_{\alpha, t_{i}}$

The value of $n_{a}$ is assigned to 1 if a methanol molecule is found in the first solvation shell at both initial $\left(t_{i}\right)$ and final $\left(t_{f}\right)$ times, and it does not leave the shell for a continuous period of time longer than $t^{*}=2 \mathrm{ps}$. To ignore transient escapes from the first solvation shell, $t^{*}$ is used. The function $\langle\cdots\rangle_{\alpha, t_{i}}$ in Eq. 6 indicates averaging over methanol molecules $(\alpha)$ and initial times $\left(t_{i}\right) . P(t)$ is approximated as the exponentially decay function $\exp \left(-t / \tau_{\mathrm{p}}\right)$ such that the residence time $\tau_{\mathrm{p}}$ can be determined as follows:

$\tau_{\mathrm{p}}=\int_{0}^{\infty} P(t) d t=\int_{0}^{\infty} \exp \left(-t / \tau_{\mathrm{p}}\right) d t$.

In this study we used $t^{*}=2.0 \mathrm{ps}$ (the value originally proposed by Impey et al. [10]). We constrained the reaction coordinate at its transition state value in the RF and GH approaches by removal of the center of mass motion of each methanol molecule that was pre-selected to form a solute pair to perform kinetics study in solution.

\section{Activation Volume}

The key parameter for determining the solvent-exchange mechanism is the activation volume, $\Delta V^{\sharp}$. It is defined as $V^{\ddagger}-V^{\text {reactant }}$, which is the difference between the partial molar volume of the transition state and that of the reactants, which can be obtained from the pressure dependence of rate constants given by Eq. 8 .

$$
\Delta V^{*}=-R T\left(\frac{\partial \ln (k)}{\partial P}\right)_{T}
$$

The pressure dependence of $\Delta V^{\ddagger}$ is small, and a linear approximation to Eq. 8 given by Eq. 9 is typically valid for solvent-exchange reactions. A negative $\Delta V^{*}$ value indicates that the solvent- 
exchange mechanism is associative, whereas a positive value indicates that the mechanism is dissociative.[6]

$\ln \left(\frac{k_{p}}{k_{0}}\right)=-\Delta V^{*} \frac{P}{R T}$

All MD simulations are performed using a modified version of the Amber9 package.[16] The polarizable force-field parameters used were those previously developed in our research group.[17] To compute PMF, a series of MD simulations are carried out at various center of mass separation distances. In each of these simulations, the center of mass separation between methanol dimer is fixed and the solvent configurations are sampled to evaluate the mean force $F(r)$. All the MD simulations are performed using the periodic boundary conditions on all three directions with a time step of 2 fs. The long-range electrostatic interactions are handled using the Ewald summation technique.[18] The SHAKE algorithm is employed in the simulations to fix the internal geometry of methanol.[19] More details of potential parameters and simulations are provided in Table 1 and in the previous publications from our group.[20]

\section{Results and Discussions}

In Figure 1a and Figure 1b, we present the computed PMFs for a methanol dimer and a water dimer in aqueous solution at three different pressures. As expected, the shapes of the free energy profiles are similar at different pressures, and the existence of well-defined contact and solvent separate pairs is obvious. In addition, the magnitude of the barrier heights to dissociation became larger as pressure increases. Moreover, with an increase in pressure, a slight left shift to smaller distances is noticed in both barrier positions. Larger well depths of the solvent separation regions at high pressures are obtained. This observation is in contrast with the computed PMFs for water dimer in solution (see Figure 1b); the data indicates that pressure 
effects on the structure of water are most important in the first hydration shell. We attribute this finding to the difference in the hydrogen-bonding network between water and methanol.

Figure 1a

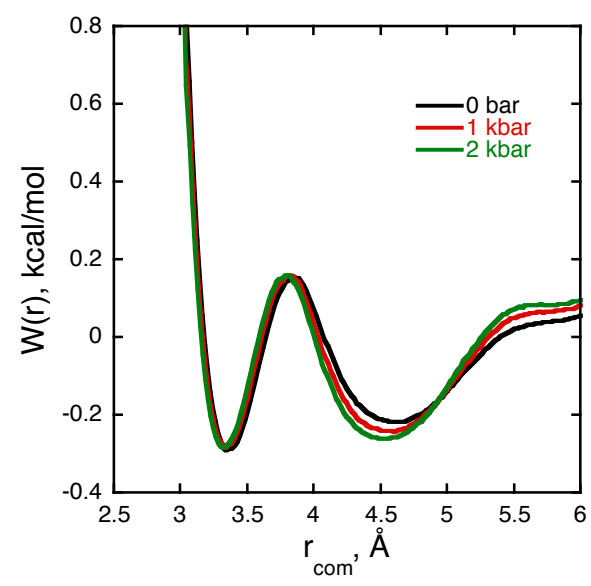

Figure 1b

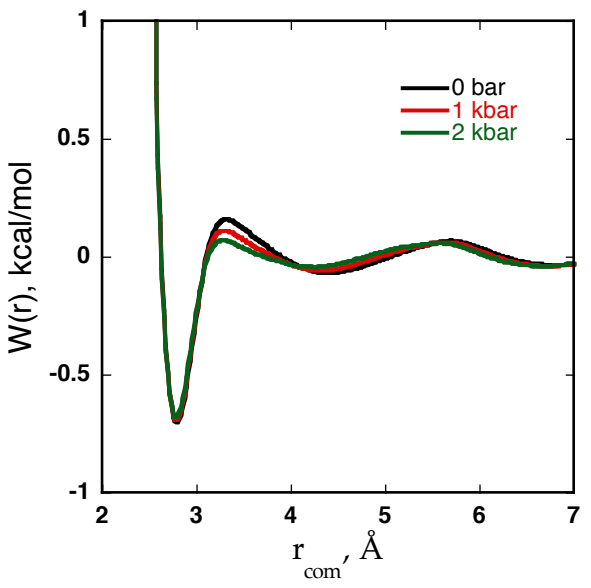

Figure 1. Computed potential of mean force, $\mathrm{W}(\mathrm{r})$, for (a) $\mathrm{MeOH}$ pair in aqueous solution as a function of pressure, and (b) for comparison, the water-water pair.

With these computed PMFs, we compute the rate constants using the TST formulism. The results for the solvent exchange computed using Eq. 3 are summarized in Table 2. As expected, because of the decrease in barrier heights, we observe an increasing trend in $k^{T S T}$ as pressure increases. The computed activation volume for this process is $-0.8 \mathrm{~cm}^{3} / \mathrm{mol}$. As discussed earlier, the negative activation volume resulting from the increase in rate constant values as pressure increases indicates an associative mechanism for the exchange of methanol molecule from the first solvation shell.

In Figure 2, we present the time-dependent transmission coefficient computed using Eq. 2 at various pressures. To compute $\kappa_{R F}(t)$, a 10-ns NVT simulation was carried out by constraining the reaction coordinate at the transition state. Simulation configurations were saved every 4 ps. Using each of these configurations, MD simulations were run both forward and backward for $2 \mathrm{ps}$ in an NVE ensemble with no constraints. The initial velocities were sampled 
through a Boltzmann distribution. The plateau value of $\kappa_{R F}$ was determined from averaging $\kappa_{R F}(t)$ over the last $0.5 \mathrm{ps}$. The same protocol at different pressures is applied for all the cases that will be discussed later in this article. The computed $\kappa_{R F}$ values as a function of pressure are reported in Table 2. Transmission coefficients are very low, indicating the existence of significant re-crossing events induced by the solvent dynamics. The value of $\kappa_{R F}$ slightly decreases as pressure increases. The activation volume obtained from the RF-method rate constants is $2.5 \mathrm{~cm}^{3} / \mathrm{mol}$, which is significantly different from the TST calculation. This finding indicates that solvent dynamics are important. As discussed earlier, the positive activation volume resulting from the decrease in rate constant values as pressure increases indicates a dissociative mechanism for the exchange of methanol molecule from the first solvation shell.

Figure 2

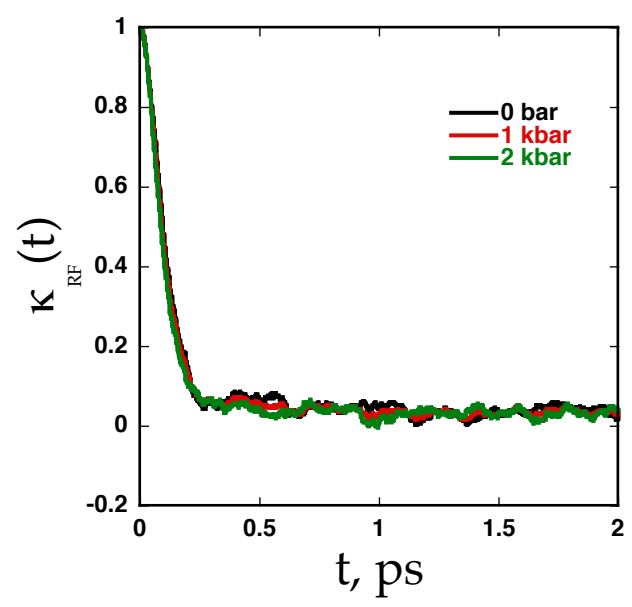

Figure 3

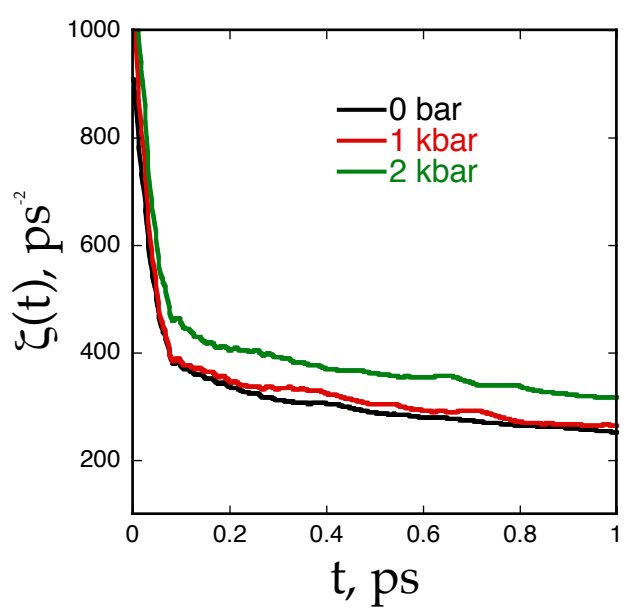

Figure 2. Computed time-dependent transmission coefficients for methanol pair as a function of pressure (left). Figure 3. Computed time-dependent kernel friction calculated for methanol pair as a function of pressure (right).

In Figure 3, we present the computed time-dependent friction kernels $\zeta(t)$ at different pressures evaluated using Eq. 3. All friction kernels show two decay time scales. Using this computed $\zeta(t)$, we evaluated the $\kappa_{G H}$ using Eq. 5, and the values at different pressure are 
reported in Table 2. We also observe that the transmission coefficients computed from GH theory decrease as pressure increases, and also, the rate constants determined from GH theory show a decreasing trend with the pressure. This observation is similar to the trend noticed when the RF method is used. A similar trend in rate constants for GH theory was observed previously in the solvent-exchange studies of liquid water. The activation volume obtained from the GH theory rate constants is $9.8 \mathrm{~cm}^{3} / \mathrm{mol}$, and the exchange process is a dissociative mechanism as found when the RF approach was used.

In Figure 4a, we show the values of $P(t)$ as the function of the pressure obtained using the IMM method. The decay of $P(t)$ is much slower for the methanol system at high pressure than at lower pressure. This would be expected because the fluid is denser at higher temperatures. This finding essentially means that the residence time, $\tau_{\mathrm{p}}$, for a methanol molecule in the first solvation shell of the other methanol; that is, $\tau_{\mathrm{p}}$ is smaller at lower pressure. This is justified quantitatively by integrating $P(t)$, which provides us with $\tau_{\mathrm{p}}=18,23$ and 34 ps for the pressure at 0,1 , and $2 \mathrm{kbar}$, respectively. In addition, we have also demonstrated that the behavior of $P(t)$ is equivalent to an exponentially decay function (i.e. $\exp \left(-\mathrm{t} / \tau_{\mathrm{p}}\right)$ ) by plotting $\ln (P(t))$ vs time. The slops of these straight lines provide us with $\tau_{\mathrm{p}}=26,36$ and $42 \mathrm{ps}$ for the pressure at 0,1 , and $2 \mathrm{kbar}$, respectively (Figure $4 \mathrm{~b}$ ). These values are higher than that of methanol exchange time scales determined using the RF method.

This discrepancy can be attributed to the arbitrary choice of the transient escape time, $t^{*}$, from the first solvation shell. However, based on the current study, there is insufficient evidence to claim that the RF method is more accurate than the IMM method.[10] The IMM approach can become sensitive to $t^{*}$, especially for low free energy barriers. D. Laage and J.T. Hynes state that a stable states picture of chemical reactions naturally discards unsuccessful exchanges and 
provides a more robust residence time calculation.[5] There is an ambiguity with the definition of the reactant and product states associated with the choice of $t^{*}$. The appeal of the IMM approach is that it is numerically effective.

Figure 4a

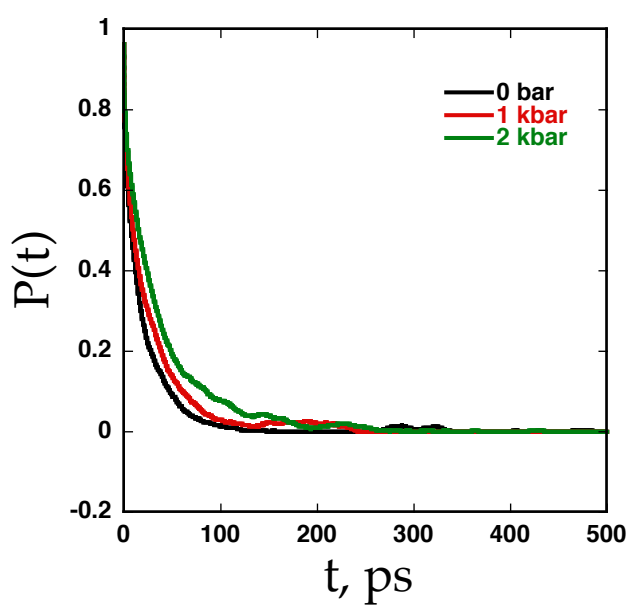

Figure $4 b$

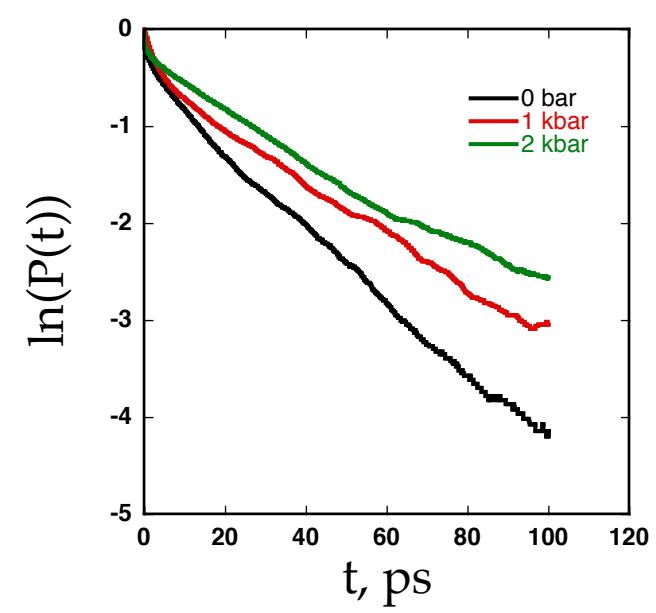

Figure 4a. Computed time-correlation function as a function of pressure used to determine the residence time of a methanol molecule is in the first solvation shell of the other methanol. Figure $4 \mathrm{~b}$ is the plot of $\ln (P(t))$ to demonstrate the behavior of $P(t)$ is equivalent to an exponentially decay $\exp \left(-\mathrm{t} / \tau_{\mathrm{p}}\right)$.

It is well known that there is the limitation of GH theory rate estimates and we explore the consequences of detailed GLE dynamics associated with this system.[21-23]

$\mu \frac{d^{2}}{d t^{2}} r(t)=-\partial_{r} W(r)-\mu \int_{0}^{t} d t^{\prime} \zeta\left(t-t^{\prime}\right) \frac{d}{d t} r\left(t^{\prime}\right)+R(t)$

$\kappa_{R F}(t)$ may be evaluated using Eq. (5), using the dynamics of the GLE.

The resulting dynamics from a harmonic approximation to the PMF gives a plateau-recrossing factor $\left(\kappa_{R F}(t)\right.$ Eq. (5)) corresponding to the GH transmission coefficient, $\kappa_{G H}$.

$W(r)=W^{\dagger}-\mu \omega_{b}^{2}\left(r-r^{\dagger}\right)^{2}$

The GLE was solved by setting up an effective Hamiltonian system consisting of the $r$ coordinate coupled to a harmonic bath. 
$H=\frac{p_{r}^{2}}{2 \mu}+W(r)+\sum_{i=1}^{N} \frac{p_{x i}^{2}}{2 \mu}+\frac{1}{2} \mu \omega_{i}^{2}\left(x_{i}-c_{i}\left(r-r^{\dagger}\right)\right)^{2}$

Where the friction is fit to a cosine expansion

$\zeta(t)=\sum_{i=1}^{N} \omega_{i}^{2} c_{i}^{2} \cos \left(\omega_{i} t\right)$

We choose a uniform distribution of frequencies $\omega_{j}=\frac{\pi}{t_{c}}(2 j-1), \mathrm{N}=100$, and $t_{c}=19.35$ psec.

This produces a numerically accurate representation of the friction and allows sufficient time for the dynamics to reach a plateau before recurrence time corresponding to the finite cosine expansion is reached. Starting from a Canonical distribution of initial conditions we evaluate the averages required for the evaluation of the reactive flux.

Figure 5

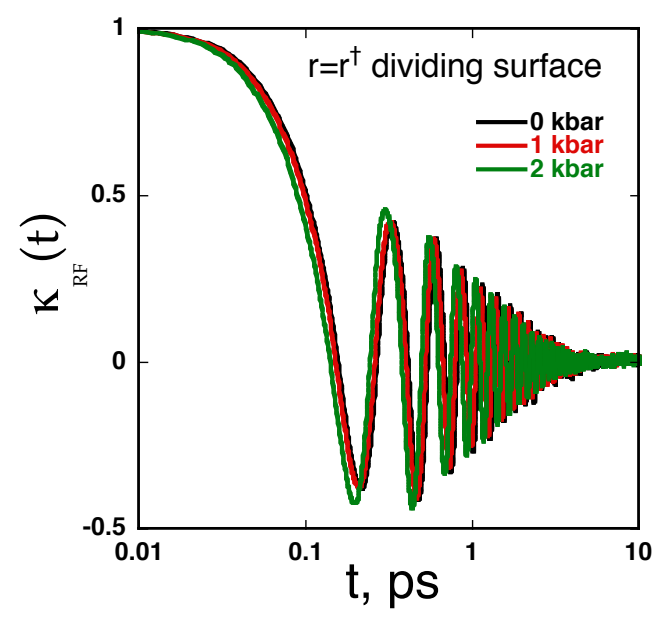

Figure 6

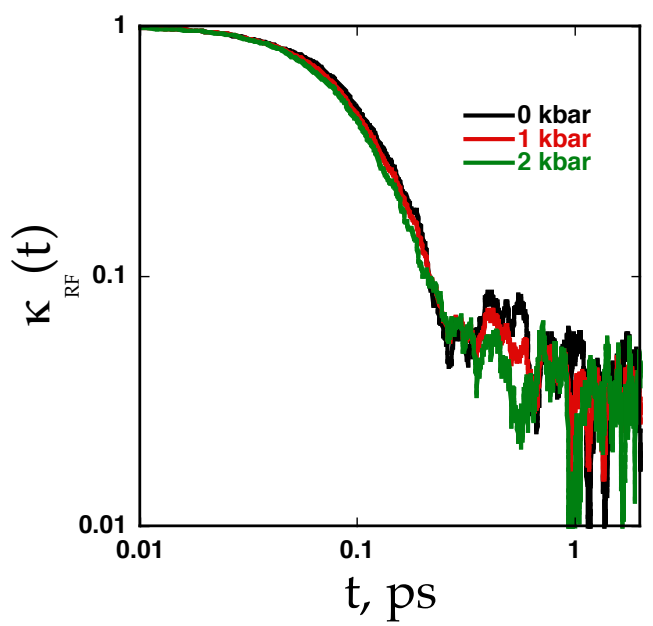

Figure 5. The resulting $\kappa(\mathrm{t})$ for the three cases of the pressure (left), Figure 6 . The short time behavior of the exact multidimensional dynamics (right).

For the case where the dividing surface separating reactants from products is described by the $\mathrm{r}$ coordinate alone, using the conventional TST dividing surface, $r=r^{\dagger}$, the resulting $\kappa_{R F}(\mathrm{t})$ are displayed in Figure 5 for the three cases of the pressure. Note the significant ringing due to multiple recrossing of the dividing surface. In Figure 6 we emphasize the short time 
behavior of the exact multidimensional dynamics. Note that the initial decay of the short time dynamics $(0.1 \mathrm{ps})$ is recovered by the GLE system, as well as the observed trend vs. pressure.

The utility of rotating a dividing surface to include bath degrees of freedom and improving TST estimates of the rate constant is well established. For the harmonic system, such a rotation will result in dynamics with no recrossing giving an exact TST estimate of the rate constant that is equal to the $\mathrm{GH}$ estimate of the rate constant. This optimal dividing surface corrsponds to the hyperplane that is orthogonal to the direction of the single unbound normal mode of the harmonic Hamiltonian expanded about $r=r^{\dagger}$.
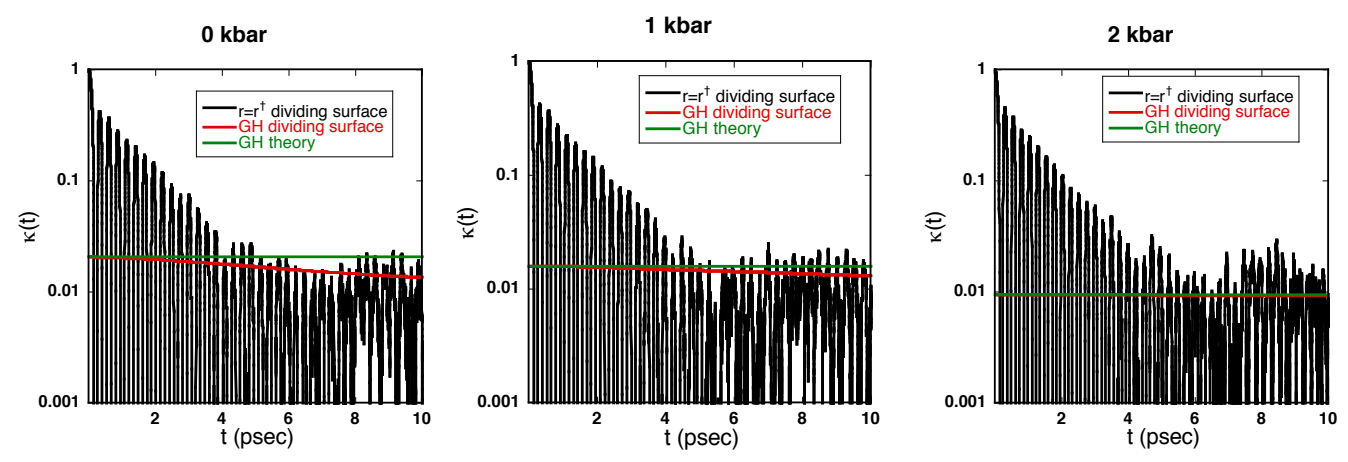

Figures 7a-7c. Transmission coefficient (recrossing factor) corresponding to GLE dynamics at 3 pressures, a) 0, b) 1 , and c) 2 kbar respectively. Dynamics launched from the conventional dividing surface (black) is compared to that of the GH hypersurface (red). Transmission coefficient corresponding to GH theory is also displayed for reference (green).

In Figures $7 \mathrm{a}-7 \mathrm{c}$ for the three pressures $(0,1 \mathrm{k}$ and $2 \mathrm{kbar})$ we compare the resulting dynamics from this dividing surface (termed "GH dividing surface") to the conventional result ( $r=r^{\dagger}$. dividing surface). The GH theory results are also displayed. From these results it is evident that the short time behavior is well described by harmonic dynamics represented by a GLE. Only for the 0 kbar case does the anharmonicity of the system significantly influence the 
resulting time dependence of the rate constant, occurring over time scales $>5 \mathrm{ps}$. The utility of launching dynamics from the GH dividing surface has also proven effective in understanding the details of ion-pair dynamics.[24] In Figure 8 the details of the long time behavior are highlighted. Resulting estimates for the rate constants are consistent with the exact dynamical results of the full system. We show in our work that a dividing surface that does not contain solvent degrees of freedom leads to numerical difficulties associated with massive dividing surface recrossing associated with strong coupling to the solvent. We find that including solvent degrees of freedom in the dividing surface results in a numerically stable estimate of the rate constant. This is possible from a GLE description of dynamics. The transfer of insight gained from the details of the GLE analysis to the fully interacting system and the choice of dividing surfaces in that system is the subject of future research. The exchange mechanism may be different when the neighbors are hydrogen-bonded or when they are in contact via their methyl groups.

In Table 3, we summary the findings of this approach along with the results obtained using GH method for comparison. We found the activation volume obtained from this method is a factor of two smaller than the corresponding GH method. We also observed that this method is closer with the results obtained by RF method.

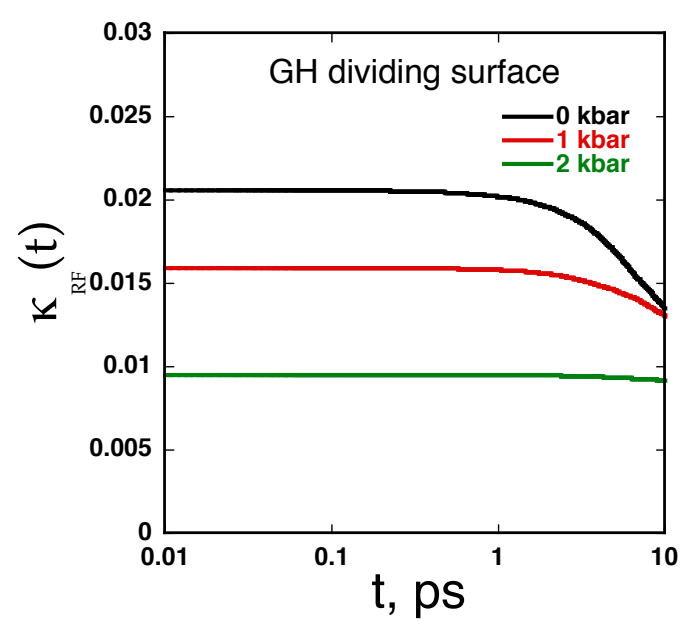


Figure 8. Dynamics launched from GH hypersurface for the three pressures. Recrossing factors from this surface correspond to $0.625,0.757$ and 0.977 for the three pressures, 0,1 , and $2 \mathrm{kbar}$ respectively. Plateau values correspond to the best estimate of the rate constant relative to conventional TST.

\section{Conclusion and Outlook}

In this article, we describe our study of the solvent exchange process in liquid methanol. We used the rate theory approach to determine the solvent-exchange rates. Also, analogous to experimental procedures, we compute pressure-dependent rates to determine the activation volume, which is a key indicator for the solvent-exchange mechanism. From our results, we observe that, because of the decrease in barrier heights, TST rate constants increase as pressure increases for all the cases. Therefore, TST results for all the cases give a negative activation volume, which is indicative of an associative mechanism. We also computed pressure-dependent rate constants using the RF and IMM methods and the GH theory, which account for re-crossings induced by solvent dynamics. In an effort to understand the nature of the dynamical recrossing and the limitation of GH theory rate estimates, we explored the consequences of detailed GLE dynamics associated with this system. We found the activation volume obtained from this method is a factor of two smaller than the GH method. We also noticed that this method agrees better with the RF method.

We recognize that the center of mass distance, as the reaction coordinate is limited. The low $\kappa$ indicates that it is not an optimal reaction coordinate. From the dynamical simulations it appears that the important reaction coordinate involves solvent rearrangement and possibly some orientation. Future work will involve decomposing the motion and the exploration of reaction coordinates involving coordination or hydrogen bond characteristics of the solvent as well as the possibility of the orientational dependence of the solutes. 
Our rate theory results do not account for the nuclear quantum effect (NQE), which plays an important role in hydrogen-bonded systems. In the past, we performed path integral Monte Carlo simulations on ion- $\left(\mathrm{H}_{2} \mathrm{O}\right)_{n}$ clusters to determine the importance of NQE on structures and to explore the quantum effects of nuclei of the clusters in the processes of hydrogen bond rearrangement.[25] We found that, because of the quantum delocalization of nuclei (especially hydrogen), the calculated quantum radial distribution functions were less structured and slightly shifted outward compared to the corresponding classical description. Path-integral techniques have been also used to investigate a variety of interesting problems. The NQE on the hydration structure of solvated excess protons in water such as tunneling, zero-point motion, and transmission coefficients were found to be significant at finite temperatures. Ongoing research efforts in our group are focused on understanding the influence of NQEs on the dynamics of ion solvation such as the water-exchange rate and the corresponding transmission coefficients using ring polymer molecular dynamics simulations.[26] 


\section{ACKNOWLEDGMENTS}

This work was supported by the U.S. Department of Energy, Office of Science, Office of Basic Energy Sciences, Division of Chemical Sciences, Geosciences, and Biosciences. The calculations were carried out using computer resources provided by the Office of Basic Energy Sciences. Discussions with Chris Mundy have enhanced this work. 


\section{REFERENCES}

1. J. T. Hynes. Chemical Reaction Dynamics in Solution, Annual Review of Physical Chemistry 36 (1985) 573.

2. E. Guardia, R. Rey, J. A. Padro, Potential of Mean Force by Constrained MolecularDynamics: A Sodium Chloride Ion-Pair in Water, Chemical Physics 155 (1991) 187.

3. G. Ciccotti, M. Ferrario, J. T. Hynes, R. Kapral, Dynamics of Ion-Pair Interconversion in a Polar-Solvent, J. Chem. Phys. 93 (1990) 7137.

4. D. A. Zichi, J. T. Hynes. A Dynamical Theory of Unimolecular Ionic Dissociation Reactions in Polar-Solvents, J. Chem. Phys. 88 (1988) 2513.

5. D. Laage, J. T. Hynes, On the Residence Time for Water in a Solute Hydration Shell: Application to Aqueous Halide Solutions, J. Phys. Chem. B 112 (2008) 7697.

6. L. Helm, A. E. Merbach, Inorganic and Bioinorganic Solvent Exchange Mechanisms. Chem. Rev. 105 (2005) 1923.

7. L. Helm, A. E. Merbach, Water Exchange on Metal Ions: Experiments and Simulations. Coord. Chem. Rev. 187 (1999) 151.

8. L. Helm, G. M. Nicolle, A. E. Merbach, Water and Proton Exchange Processes on Metalions. Advances in Inorganic Chemistry - Including Bioinorganic Studies, 57 (2005) 327.

10. R. W. Impey, P. A. Madden, I. R. Mcdonald, Hydration and Mobility of Ions in Solution. J. Phys. Chem. 87 (1983) 5071.

11. D. Chandler, Statistical-Mechanics of Isomerization Dynamics in Liquids and Transition-State Approximation. J. Chem. Phys. 68 (1978) 2959.

12. R. F. Grote, J. T. Hynes, The Stable States Picture of Chemical-Reactions 2. Rate Constants for Condensed and Gas-Phase Reaction Models. J. Chem. Phys. 73 (1980) 2715.

13. D. G. Truhlar, W. L. Hase, J. T. Hynes, Current Status of Transition-State Theory. J. Phys. Chem. 87 (1983) 2664.

14. E. Pollak, Theory of Activated Rate Processes: A New Derivation of Kramers' Expression. J. Chem. Phys. 85 (1986) 865.

15. D. G. Truhlar and B. C. Garrett, "Variational Transition State Theory," Annual Review of Physical Chemistry, Vol. 35, edited by B. S. Rabinovitch, J. M. Schurr, and H. L. Strauss (Annual Reviews, Inc., Palo Alto, California, 1984), pp. 159-189.

16. Case, D. A.; Darden, T. A.; Cheatham III, T. E.; Simmerling, C. L.; Wang, J.; Duke, R. E.; Luo, R.; Merz, K. M.; Pearlman, D. A.; Crowley, M.; Walker, R. C.; Zhang, W.; Wang, B.; Hayik, S.; Roitberg, A.; Seabra, G.; Wong, K. F.; Paesani, F.; Wu, X.; Brozell, S.; Tsui, V.; Gohlke, H.; Yang, L.; Tan, C.; Mongan, J.; Hornak, V.; Cui, G.; Beroza, P.; Mathews, D. H.; Schafmeister, C.; Ross, W. S.; Kollman, P. A., Amber 9. University of California, San Francisco: 2006.

17. L. X. Dang, T. M. Chang, Many-Body Interactions in Liquid Methanol and Its Liquid/Vapor Interface: A Molecular Dynamics Study. J. Chem. Phys. 119 (2003) 9851.

18. U. Essmann, L. Perera, M. L. Berkowitz, T. Darden, H. Lee, L. G. Pedersen, A Smooth Particle Mesh Ewald Method. J. Chem. Phys. 103 (1995) 8577.

19. J. P. Ryckaert, G. Ciccotti, H. J. C. Berendsen, Numerical-Integration of Cartesian Equations of Motion of a System with Constraints Molecular Dynamics of N-Alkanes. J. Comp. Phys. 23 (1977) 327. 
20. H. V. Annapureddy, L. X. Dang, Understanding the Rates and Molecular Mechanism of Water-Exchange around Aqueous Ions Using Molecular Simulations. J. Phys. Chem. B 118 (2014) 8917.

21. G. K. Schenter, R. P. McRae, B. C. Garrett, Dynamic Solvent Effects on Activated Chemical-Reactions .1. Classical Effects of Reaction-Path Curvature. J. Chem. Phys. 97 (1992) 9116.

22. R. P. McRae, G. K. Schenter, B. C. Garrett, Dynamic Solvent Effects on Activated Chemical Reactions 2. Quantum Mechanical Effects. J. Chem. Soc. Farad. Trans. 93 (1997) 997.

23. B. C. Garrett, G. K. Schenter, Variational Transition-State Theory for Activated Chemical-Reactions in Solution. Int. Rev. Phys. Chem. 13 (1994) 263.

24. E. Pluharova, M. D. Baer, G. K. Schenter, P. Jungwirth, and C. J. Mundy]. The Dependence of the Rate of LiF Ion Pairing on the Description of Molecular Interaction, J. Phys. Chem. B (In press).

25. Huadong Gai, Gregory K. Schenter, Liem X. Dang and Bruce C. Garrett, "Quantum Simulation of Ion-Water Cluster $\mathrm{I}^{-}\left(\mathrm{H}_{2} \mathrm{O}\right)_{\mathrm{n}}$ : The Importance of Quantum Effects and Anharmonicity", J. Chem. Phys. 104 (1996) 7502.

26. S. Habershon, D. E. Manolopoulos, T. E. Markland and T. F. Miller III, Annu. Rev. Phys. Chem. 64 (2013) 387. 
Table 1. Optimized potential parameters for methanol used in the MD simulation.

\begin{tabular}{|c|c|c|c|c|}
\hline Atom type & $\sigma(\AA)^{\mathrm{a}}$ & $\varepsilon(\mathrm{kcal} / \mathrm{mol})^{\mathrm{a}}$ & $q(\mathrm{e})^{\mathrm{b}}$ & $\alpha\left(\AA^{3}\right)^{\mathrm{c}}$ \\
\hline $\mathrm{O}$ & 3.2340 & 0.1825 & -0.4770 & 2.02 \\
\hline $\mathrm{HO}$ & 0.0000 & 0.0000 & 0.3361 & 0.00 \\
\hline CT & 3.3854 & 0.1300 & -0.0954 & 0.00 \\
\hline HT & 2.5034 & 0.0230 & 0.0787 & 0.00 \\
\hline
\end{tabular}

Table 2. Computed activation barriers and rate coefficients as a function of pressure at $300 \mathrm{~K}$.

\begin{tabular}{|c|c|c|c|c|c|}
\hline $\begin{array}{c}\text { Pressure } \\
(\text { bar })\end{array}$ & $\begin{array}{c}\text { Barrier Height } \\
(\mathrm{kcal} / \mathrm{mol})\end{array}$ & $k^{T S T}\left(\mathrm{ps}^{-1}\right)$ & $\kappa_{\mathrm{RF}} \mathrm{k}^{\mathrm{TST}}$ & $\kappa_{\mathrm{GH}} \mathrm{k}^{\mathrm{TST}}$ & $\kappa_{\mathrm{IMM}}$ \\
\hline 0 & $0.45 \pm 0.06$ & $1.67 \pm 0.21$ & $0.070 \pm 0.017$ & $0.020 \pm 0.003$ & $0.056 \pm 0.070$ \\
\hline 1,000 & $0.44 \pm 0.06$ & $1.73 \pm 0.21$ & $0.060 \pm 0.016$ & $0.016 \pm 0.002$ & $0.043 \pm 0.005$ \\
\hline 2,000 & $0.44 \pm 0.06$ & $1.79 \pm 0.22$ & $0.570 \pm 0.015$ & $0.010 \pm 0.001$ & $0.029 \pm 0.004$ \\
\hline$\Delta \mathrm{V}^{\ddagger}$ & & $\begin{array}{c}-0.8 \pm 0.1 \\
\mathrm{~cm}^{3} / \mathrm{mol}\end{array}$ & $\begin{array}{c}2.5 \pm 0.3 \\
\mathrm{~cm}^{3} / \mathrm{mol}\end{array}$ & $\begin{array}{c}9.2 \pm 1.2 \\
\mathrm{~cm}^{3} / \mathrm{mol}\end{array}$ & $\begin{array}{c}8.0 \pm 1.0 \\
\mathrm{~cm}^{3} / \mathrm{mol}^{3}\end{array}$ \\
\hline
\end{tabular}

Table 3. Comparison between GH and GLE methods

\begin{tabular}{|c|c|c|}
\hline $\begin{array}{c}\text { Pressure } \\
\text { (bar) }\end{array}$ & $\kappa_{\mathrm{GH}} \mathrm{k}^{\mathrm{TST}}$ & $\kappa_{\mathrm{GLE}} \mathrm{k}^{\mathrm{TST}}$ \\
\hline 0 & $0.020 \pm 0.003$ & $0.013 \pm 0.002$ \\
\hline 1,000 & $0.016 \pm 0.002$ & $0.012 \pm 0.002$ \\
\hline 2,000 & $0.010 \pm 0.001$ & $0.010 \pm 0.001$ \\
\hline$\Delta \mathrm{V}^{*}$ & $\begin{array}{c}9.2 \pm 1.2 \\
\mathrm{~cm}^{3} / \mathrm{mol}\end{array}$ & $\begin{array}{c}4.6 \pm 0.6 \\
\mathrm{~cm}^{3} / \mathrm{mol}\end{array}$ \\
\hline
\end{tabular}




\section{Biography}

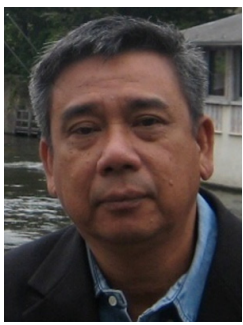

Dr. Liem X. Dang is a Scientist in the Physical Chemistry Division at the Pacific Northwest National Laboratory (PNNL), where he has been since 1991 . He received his BS from Florida Institute of Technology in 1980 and a Ph.D. from the University of California, Irvine in 1985 . He was a postdoctoral fellow at the University of California, San Francisco and Visiting Scientist IBM Almaden Research Center, CA before joining the staff at PNNL in 1991. Presently, He is also an Adjunct Professor at The School of Chemical Engineering, University of Queensland, Australia and at the Department of Metallurgical Engineering, College of Mines and Earth Sciences, University of Utah, USA. Among his honors, He is a Fellow of the American Physical Society and of the American Association for the Advancement of Science and is also on the Editorial Board for the Journal of Physical Chemistry $A / B / C$ and Letters.

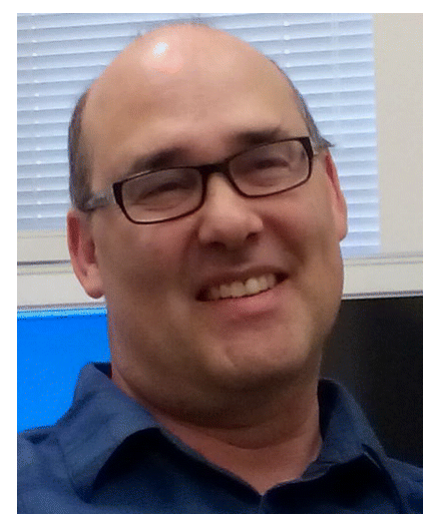

Dr. Gregory Schenter has been with Pacific Northwest National Laboratory (PNNL) since 1988 and was named a Laboratory Fellow in 2009. Dr. Schenter's research focuses on the development of statistical mechanical techniques to better understand molecular interactions and molecular processes in complex condensed-phase systems. Schenter received his Ph.D. in Applied Physics from Cornell University in 1988. 


\section{Table of Contents}
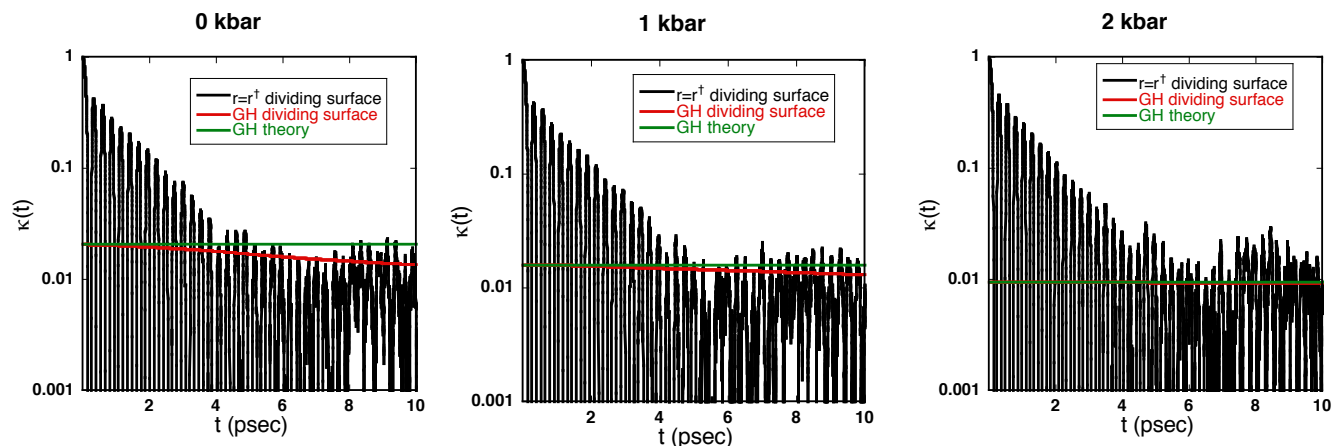
Proposal Cover Art
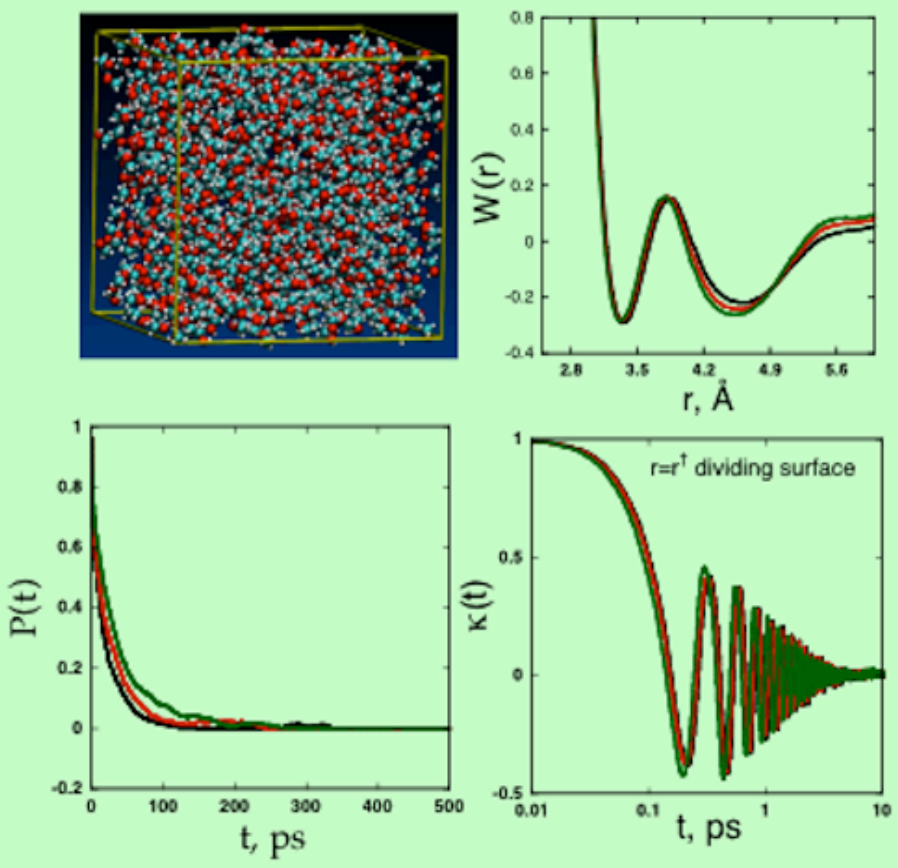